\title{
Evolutionary dynamics in fluctuating environment
}

\author{
Immanuel Meyer $\odot$ and Nadav M. Shnerb $\odot$ \\ Department of Physics, Bar-Ilan University, Ramat-Gan IL52900, Israel
}

(Received 16 September 2019; accepted 4 May 2020; published 9 June 2020)

\begin{abstract}
Temporal environmental variations are ubiquitous in nature, yet most of the theoretical works in population genetics and evolution assume fixed environment. Here we analyze the effect of variations in selection sign, selection intensity, and population size on the fate of a mutant type. Using Kimura's diffusion approximation we present simple formulas for effective population size and effective selection, and use it to calculate the chance of ultimate fixation, the time to fixation, and the time to absorption (either fixation or loss). For simple models, in which the number of environmental states is relatively small, the effective parameters are obtained analytically. For more complicated models, where the monitoring of the weights of all microstates is complicated, we present a semianalytic solution whose parameters are obtained from short numerical experiments. Our analysis shows perfect agreement with numerical solutions for neutral, beneficial, and deleterious mutant, under periodic and stochastic environmental variations and different competition modes.
\end{abstract}

DOI: 10.1103/PhysRevResearch.2.023308

\section{INTRODUCTION}

Evolution takes place in a temporally fluctuating environment, and the interplay between the deterministic effect of selection and these temporal variations poses a major theoretical challenge. Even if the macroproperties of the environment are fixed over time, local fluctuations affect the reproductive success of individuals in an uncorrelated manner, a phenomenon known as genetic drift, demographic stochasticity, or internal noise. These uncorrelated fluctuations generate, for a population of size $n$, an $O(\sqrt{n})$ abundance variations. Moreover, in many cases entire populations are subject to macroenvironment variations that affect coherently the reproductive success (birth and death rates) of all individuals. These effects may be either periodic (seasonality) or stochastic (draughts, floods, precipitation fluctuations, and so on) and yield stronger, $O(n)$, abundance variations. In the literature, stochastic temporal variations of this kind are known as environmental stochasticity or external noise [1].

Traditionally, the theory of population genetics and evolution was focused on the interference of selection (with intensity $s$ ) and demographic stochasticity (drift) [2], assuming fixed birth and death rates. Effects of environmental variations were considered only rarely [3,4]. Recent empirical studies have documented periodic and stochastic coherent variations in relative fitness [5-7] as well as variations in the birth and death rates [8-14]. These findings triggered a renewed interest in the effect of macroenvironmental variations on evolutionary dynamics [15-22].

Published by the American Physical Society under the terms of the Creative Commons Attribution 4.0 International license. Further distribution of this work must maintain attribution to the author(s) and the published article's title, journal citation, and DOI.
When $n$ is large, macroenvironmental fluctuations yield stronger abundance variations and their effect dominates the noise associated with genetic drift, but when $n$ is small drift effects are dominant. In particular, biological extinction is related to the discreetness of individuals which manifests itself only via the drift. Therefore, the small $n$ sector has crucial importance to evolutionary dynamics. Practical implications require one to take into account both drift and correlated variations, which makes the theoretical analysis quite intricate.

The simplest and most important scenario in evolution involves zero-sum competition between two haploid types (a very similar model describes the dynamics of a two-allele, one locus system for diploid population with additive effect on fitness). The total number of individuals is $N$, and the frequency of a given species with abundance $n$ is $x=n / N$. The three properties that govern evolutionary dynamics are $\Pi(x)$, the chance that a mutant type will reach ultimate fixation, $T_{A}(x)$, the time to absorption (either fixation or loss), and $T_{F}(x)$, the time to fixation. $\Pi(x)$ plays a determinant role in the evolutionary dynamics as it controls the long-term adaptation of populations and the rate of accumulation of neutral substitutions (molecular clock) [23]. $T_{A}(x)$ sets the timescale for coexistence and controls the crossover from successive fixation to clonal interference dynamics [24], and $T_{F}(x)$ governs the adaptation process and the speed of evolution [25].

Here we present a generic scheme for the analysis of this system when both $s$ and $N$ vary in time in an arbitrary (stochastic or periodic) manner. Our analysis relays on three assumptions.

(1) The selection parameter $s$ is assumed to be small and $O\left(s^{2}\right)$ terms are neglected.

(2) The system is in its "microevolutionary" (annealed) regime as defined in [15]: the time to fixation is assumed to be large enough to allow for a reliable sampling of the state space by a typical trajectory. 
(3) We implement the diffusion approximation, which requires the relevant quantities (like $\Pi$ ) to be smooth enough over the integers [26].

These assumptions are quite plausible. Empirical selection parameters are usually much smaller than one; for example, selective sweeps in the long-term evolutionary experiment of Lenski and co-workers reach fixation in about 1000 generations [27], suggesting $s \approx 0.001$. Such a timescale is much larger than the correlation time of many environmental variations, and $s^{2}$ terms are indeed tiny. The implementation of the diffusion approximation is natural in the regime where $s$ is small and $N$ is large. This approximation is known to be remarkably robust to modifications of the microscopic dynamics of the model [2].

Given assumptions (1)-(3), we will show that the standard formulas, that were obtained many years ago for systems with fixed $N$ and $s$ [23,28], are applicable in temporally varying environment, provided that $N$ and $s$ are replaced by effective values $N_{\text {eff }}$ and $s_{\text {eff }}$. For any given state of the external parameters, the frequency $x$ may change, $x \rightarrow x+\Delta x$, and the emerging diffusion (or backward Kolmogorov) equations depend only on the mean and the variance of these changes. $s_{\text {eff }}$ is the total selection, i.e., the weighted contribution of all environmental states to the mean of $\Delta x$, while $N_{\text {eff }}$ is determined by the weighted contribution to the variance of $\Delta x$.

Importantly, when our assumptions hold the contributions of different microstates are commutative, i.e., the order of events does not matter, only their rate of occurrence. Accordingly, periodic and stochastic variations yield the same values of $s_{\text {eff }}$ and $N_{\text {eff }}$, provided that they visit, on average, the same microstates in the same rates. This feature is exploited through this paper, as it allows us to choose the simplest setup for our analytic calculations.

This paper is organized as follows. In the next section we present our analysis using simple models that allow for complete analytic derivation of $N_{\text {eff }}$ and $s_{\text {eff }}$, and then compare the predictions to the outcomes of numerical simulations. In Sec. III we treat the general case, where a fully analytic solution is too cumbersome. For that case we implement a semianalytic approach, in which the effective parameters are derived from an easy and quick numerical simulation of a single environmental cycle. Technical details of the calculations and the numerics are given in the Appendixes. A general overview is presented in the Discussion section.

\section{ANALYTIC SOLUTION OF MODELS WITH SHARP ENVIRONMENTAL TRANSITIONS}

In this section we present a solution for evolution in fluctuating environment, provided that the system jumps abruptly between two environmental states. This class of models allow for a fully analytic solution and provides good insights into the general problem. They also allows us to demonstrate the robustness of the results and the periodic-stochastic equivalence, thus laying the foundations for Sec. III, where a semianalytic approach is implemented in the general case.

\section{A. Models definition}

We consider a wild type and a mutant population competing for a single resource (say, food). The relative fitness of the mutant type, $s$, reflects its access ability to consume or to reach the food. The parameter $s$ is positive for a beneficial mutant, negative for a deleterious mutant, and $s=0$ for a neutral mutant. Under fixed environmental conditions, the absolute value of $s N$ sets the strength of selection: when $|s N|<1$ (weak selection) demographic fluctuations (drift) dominate and the dynamics is effectively neutral; when $|s N|>1$ (strong selection) selective forces dominate. For fixed $N$ and $s$ the solutions for these quantities are known for many years [23,28].

We allow the total size of the community, $N$, to vary in time due to seasonal fluctuations (such as food shortages during winter) or stochastic disturbances (droughts, floods). The carrying capacity decreases when the environment deteriorates and increases when the environmental conditions improve. Fluctuations in selective parameters may be correlated or uncorrelated with fluctuations in population size, and the general theory presented in Sec. III allows one to deal with the general situation once $s(N, t)$ is given. For the sake of concreteness we consider here three types of selective effects, one (characterized by the parameter $s$ ) at population equilibrium ( $N$ fixed) and two others during periods of growth $\left(s_{g}\right)$ or decline $\left(s_{d}\right)$ in $N$. These parameters may differ in amplitude and in sign: for example, plants with larger seeds that admit larger metabolic reserves may have a better chance of establishment during periods of increasing stress, while smaller seeds, produced in larger quantities, have a better chance to colonize a new suitable habitat during fast expansion [29].

In this section we model resource variations that affect the total population size $N$ through a simple two-state dynamics. When the resource density declines, the total size decreases from $N$ to $r N$ (without loss of generality we assume $r<$ 1 ), while an increase in the amount of available resource is followed by population growth from $r N$ back to $N$ (see Fig. 1). Population size variations are assumed (in this section) to be instantaneous.

The persistence time of the environment is $\tau$. Two types of dynamics, periodic or stochastic, are considered.

(i) In periodically varying environment (seasonal variations) $\tau$ is the duration between two successive switches.

(ii) In randomly fluctuating environment, the time between two successive switches is drawn from an exponential distribution with mean $\tau$.

At equilibrium, competition takes place in a series of birthdeath events, and time is incremented by $1 / N(t)$ after each of these events, so $\tau$ is measured in units of a generation (one generation $=N$ elementary birth-death events).

We consider two types of zero-sum equilibrium dynamics: local and global.

(i) The local dynamics corresponds to the case where a random encounter between individuals may involve a fight for a piece of food, a mate, or a territory. To model that, two individuals are picked at random for a "duel"; the loser dies and the winner produces a single offspring. If the mutant frequency is $x$, the chance of a duel between a mutant and a wild type is $2 x(1-x)$. The chance of the mutant to win the duel is defined to be $1 / 2+s / 4$, so $s$ reflects the intensity of selection. When $s=0$ the equilibrium dynamics is neutral. 


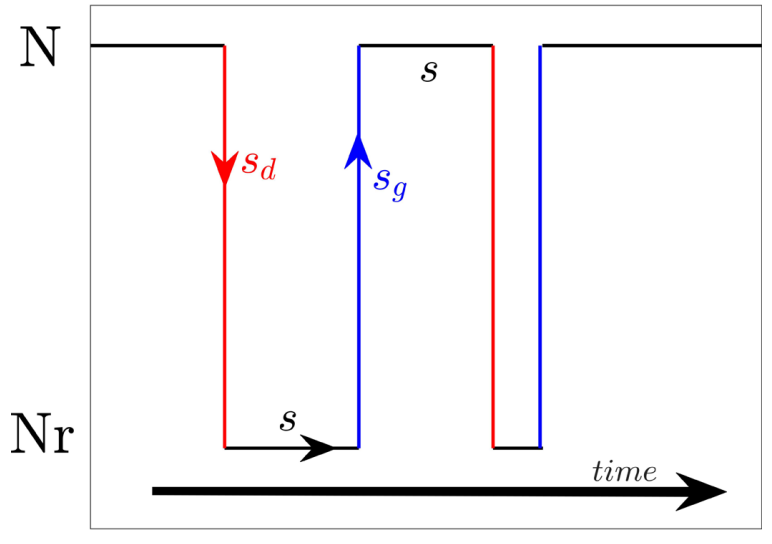

FIG. 1. Illustration of population size dynamics in varying environment. Under high resource density the total number of individuals is $N$, while during periods of shortage the total number is $r N$. Transitions between the two states correspond to periods of sharp population growth or sharp decline, here taken to be instantaneous. If the dynamics is periodic, the time between two consecutive jumps (between sharp growth to sharp decline or vise versa) is $\tau$ generations. If the dynamics is stochastic (as in this cartoon) the persistence time is picked from an exponential distribution with mean $\tau$. Selection parameter at equilibrium is $s, s_{g}$ is the selection parameter during periods of sharp growth, and $s_{d}$ is the selection parameter during sharp decline.

(ii) Global dynamics best illustrates the competition in a forest, say, where an adult tree dies at random and the gap is recruited by a single seed or seedling. If the local seed bank reflects the composition of the whole forest (long distance dispersal), the chance of the mutant type to capture the gap depends on both its abundance and its fitness. In our global model this chance is

$$
\frac{x e^{s}}{1-x+x e^{s}}
$$

where the fitness factor $e^{s}$ reflects an excess productivity of seeds or the excess chance of germination per seed.

With this parametrization, both models yield, to the leading order in $s$, the logistic behavior [21]

$$
\dot{x}=\operatorname{sx}(1-x) \text {. }
$$

As explained below, this allows us to implement the same formulas for the effective population size and the effective selection in both cases, once the $s$ value is equal.

During periods of sharp growth $(1-r) N$ slots open up, and the number of new recruits by the mutant strain is picked at random from $B_{N(1-r)}\left[x+s_{g} x(1-x)\right]$, a binomial distribution with $N(1-r)$ trials where the chance to win each trial is

$$
\frac{x e^{s_{g}}}{x e^{s_{g}}+(1-x)} \approx x+s_{g} x(1-x) .
$$

Accordingly, in a period of sharp growth on average $x \rightarrow x+$ $s_{g} x(1-x)(1-r)$ and the leading contribution to the variance is $\operatorname{Var}(x)=x(1-x)(1-r) / N$.

During a period of sharp decline, each individual survives with a certain probability that may depend on its phenotype. To model that, we assumed the number of mutant survivors to
TABLE I. Glossary.

\begin{tabular}{lc}
\hline \hline Sharp transition model & \\
\hline$N$ & Total number of individuals \\
$n($ or $m)$ & Number of individuals of focal species \\
$x=n / N$ & Focal species' frequency \\
$\tau$ & Average persistence time of the environment \\
$s$ & Selection parameter at equilibrium \\
$s_{g}$ & Selection parameter during growth \\
$s_{d}$ & Selection parameter during decline \\
$r$ & Ratio between high and low population states \\
Logistic model (Sec. III) & \\
$K_{+} K_{-}$ & Maximum/minimum carrying capacity \\
$\lambda$ & Total population's growth rate \\
\hline \hline
\end{tabular}

be picked from $B_{x N}\left[r\left(1+s_{d}\right)\right]$ and the number of wild type survivors is drawn from $B_{(1-x) N}[r]$ [of course the condition $s_{d} \leqslant(1-r) / r$ must be imposed]. If $s_{d} \ll 1$, in a period of sharp decline $x \rightarrow x+s_{d} x(1-x)$ and $\operatorname{Var}(x)=x(1-x)(1-$ $r) /(N r)[30]$.

Sort descriptions of the various parameters used through this paper are presented in Table I.

\section{B. Effective population size and effective selection}

Under purely demographic stochasticity, when population size is fixed at $N$ and the mutant strain has fixed log fitness $s$ and frequency $x=n / N$, the chance of fixation $\Pi(x)$ is known to satisfy $[23,28]$

$$
\frac{1}{2 N^{2}} \Pi^{\prime \prime}(x)+\frac{s}{2 N} \Pi^{\prime}(x)=0,
$$

with the boundary conditions $\Pi(0)=0$ and $\Pi(1)=1$. Similarly, the time to absorption $T_{A}$ satisfies

$$
\frac{1}{2 N^{2}} T_{A}^{\prime \prime}(x)+\frac{s}{2 N} T_{A}^{\prime}(x)=-\frac{1}{N x(1-x)},
$$

with $T_{A}(0)=T_{A}(1)=0$.

The solution for $\Pi(x)$ is a known formula,

$$
\Pi(x)=\frac{1-e^{-N s x}}{1-e^{-N s}} .
$$

An important parameter is the strength of selection $s N$, which is the ratio between selection intensity and the strength of the drift (the variance of $x$ variations per generation $1 / N$ ). As mentioned above, when $|s N| \ll 1$ (weak selection) the process is effectively neutral, the sign of $s$ is irrelevant, and $\Pi(x) \approx x$. If $|s N| \gg 1$ (strong selection) the chance of a single deleterious mutant $(x=1 / N)$ to reach fixation decays exponentially with $s N$, while for a single beneficial mutant $(s>0) \Pi(1 / N) \approx s$.

Now let us implement the diffusion approximation in varying environment. Specifically we would like to consider stochastic environmental variations with local competition at equilibrium. Below we explain why the outcome is applicable to the other cases (global competition and periodic variations).

In our model the population size flips instantaneously between its two allowed values, $N$ and $r N$, so during each elementary time step either the system jumps $(N \leftrightarrow r N)$ or 
a duel between two individuals takes place. Accordingly, $J^{+}=1 / N \tau$ and $J^{-}=1 / N r \tau$ are the chances, per elementary competition step (duel), that the environment flips to the other state.

When a duel takes place, it will be an intraspecific duel with probability $1-2 x(1-x)$ and an interspecific duel with probability $2 x(1-x)$. Intraspecific duels have no effect on the number of mutant individuals (either a wild type replaces a wild type or a mutant replaces a mutant). After an interspecific duel, the number of mutants grows by one with probability $1 / 2+s / 4$ or decreases by one with probability $1 / 2-s / 4$.

When the environmental conditions suddenly improve, $r N \rightarrow N$, the overall population increases by $N(1-r)$ individuals. As explained, the share of the mutant species is taken to be $B_{N(1-r)}\left[x+s_{g} x(1-x)\right]$, so the chance of the mutant type to grow from $n$ individuals to $m$ individuals is

$$
P_{n \rightarrow m}^{\text {growth }}=f_{N(1-r), x+s_{g} x(1-x)}(m-n), \quad m \geqslant n,
$$

where $f_{b, p}(a)$ is the probability mass function of the binomial distribution, i.e., the chance to pick $a$ successes from $b$ trials with success probability $p$. By the same token $P_{n \rightarrow m}^{\text {growth }}$ describes the chance of frequency change from $x=n / N$ to $x^{\prime}=m / N$.

During periods of sharp decline, on average $N \rightarrow r N$. If $s$ is small, this may be achieved if each wild type individual survives with probability $r$ and each mutant individual survived with probability $r\left(1+s_{d}\right)$. Accordingly, if the number of mutant individuals before decline is $n$, the number after decline is picked from $B_{n}\left[r\left(1+s_{d}\right)\right]$, and correspondingly the number of wild type individuals after the decline is $B_{N-n}[r]$. Therefore, for the mutant,

$$
P_{n \rightarrow m}^{\text {decline }}=f_{n, r\left(1+s_{d}\right)}(m)
$$

and for the wild type

$$
P_{N-n \rightarrow m^{\prime}}^{\text {decline }}=f_{N-n, r}\left(m^{\prime}\right) .
$$

The chance to reach $x^{\prime}=m /\left(m+m^{\prime}\right)$, starting from $x=n / N$, is

$$
P_{x \rightarrow x^{\prime}}^{\text {decline }}=f_{n, r\left(1+s_{d}\right)}(m) f_{N-n, r}\left(m^{\prime}\right) .
$$

To compare our analytic expression with Markov-matrixbased numerics that require exactly $r N$ individuals in the poor state we implemented a slightly different decline procedure that has the same mean and variance, while in Monte Carlo simulations we used the two binomial deviates. Details are given in Appendix B.

Overall, the transition probabilities in each elementary time step are

$$
\begin{aligned}
W_{x \rightarrow x}^{ \pm} & =\left(1-J^{ \pm}\right)[1-2 x(1-x)], \\
W_{x \rightarrow x+1 / N}^{+} & =\left(1-J^{+}\right) 2 x(1-x)\left(\frac{1}{2}+\frac{s}{4}\right), \\
W_{x \rightarrow x+1 /(r N)}^{-} & =\left(1-J^{-}\right) 2 x(1-x)\left(\frac{1}{2}+\frac{s}{4}\right), \\
W_{x \rightarrow x-1 / N}^{+} & =\left(1-J^{+}\right) 2 x(1-x)\left(\frac{1}{2}-\frac{s}{4}\right), \\
W_{x \rightarrow x-1 /(r N)}^{-} & =\left(1-J^{-}\right) 2 x(1-x)\left(\frac{1}{2}-\frac{s}{4}\right),
\end{aligned}
$$

$$
\begin{aligned}
& Q_{x \rightarrow x^{\prime}}^{-}=J^{+} P_{x \rightarrow x^{\prime}}^{\text {decline }}, \\
& Q_{x \rightarrow x^{\prime}}^{+}=J^{-} P_{x \rightarrow x^{\prime}}^{\text {growth }} .
\end{aligned}
$$

In these expressions $W_{x \rightarrow x^{\prime}}^{ \pm}$(or equivalently $W_{n \rightarrow n_{1}}^{ \pm}$) is the chance to reach $x^{\prime}$ from $x$ in an elementary duel when the environment is poor (minus, total population $r N$ ) or rich (plus, total population $N$ ). Correspondingly, $Q_{x \rightarrow x^{\prime}}^{ \pm}$(or equivalently $Q_{n \rightarrow m}^{ \pm}$) is the chance to reach $x^{\prime}$ if the system was at $x$ right before a sharp decline or a sharp increase.

If at $t=0$ the mutant strain is represented by $n$ individuals, and the environment is in its rich/poor (plus/minus) state, the chance of fixation by the mutant, $\Pi_{n}^{ \pm}$, satisfies the discrete backward Kolmogorov equation (BKE),

$$
\begin{aligned}
\Pi_{n}^{ \pm}= & W_{n \rightarrow n+1}^{ \pm} \Pi_{n+1}^{ \pm}+W_{n \rightarrow n-1}^{ \pm} \Pi_{n-1}^{ \pm}+W_{n \rightarrow n}^{ \pm} \Pi_{n}^{ \pm} \\
& +\sum_{m} Q_{n \rightarrow m}^{\mp} \Pi_{m}^{\mp} .
\end{aligned}
$$

This BKE is a $(N+r N-2) \times(N+r N-2)$ linear system and may be solved numerically to obtain $\Pi_{n}^{ \pm}$. This matrixbased technique was used to obtain the numerical results of Figs. 2 and 3; see Appendix B.

To solve the problem analytically, one would like to map the difference equation (11) into a differential equation. We define a significant step as an interspecific duel (note that $n$ must change in such a significant step). For a given $x$, the chance of an elementary step (duel) to be significant is $2 x(1-$ $x$ ). The mean number of elementary steps needed for two sharp jumps (an increase and the following decrease or vice versa) is $(N+r N) \tau=N(1+r) \tau$. Therefore, the number of significant steps per a single decline/increase jump is

$$
\frac{1}{\eta} \equiv x(1-x) N \tau(1+r) .
$$

The chance of a single event to be a jump is therefore $\eta /(1+$ $\eta$ ) and its chance to be a competition step (duel) is $1 /(1+\eta)$. Moreover, for each significant step (interspecific duel) in the rich environment there are only $r$ significant steps in the poor environment.

Now we can write the backward Kolmogorov equation for $\Pi(x) \equiv\left[\Pi^{+}(x)+\Pi^{-}(x)\right] / 2$, i.e., for the chance to reach fixation when the initial state is in the plus or in the minus state with probability $1 / 2$. When written per significant step it takes the form

$$
\begin{aligned}
\Pi(x)= & \frac{1}{1+\eta}\left[A\left(\frac{1}{2}+\frac{s}{4}\right) \Pi(x+1 / N)+A\left(\frac{1}{2}-\frac{s}{4}\right)\right. \\
& \times \Pi(x-1 / N)+B\left(\frac{1}{2}+\frac{s}{4}\right) \Pi(x+1 / r N) \\
& \left.+B\left(\frac{1}{2}-\frac{s}{4}\right) \Pi(x-1 / r N)\right] \\
& +\frac{\eta}{2(1+\eta)} \sum_{x^{\prime}}\left[P_{x \rightarrow x^{\prime}}^{\text {growth }} \Pi\left(x^{\prime}\right)+P_{x \rightarrow x^{\prime}}^{\text {decline }} \Pi\left(x^{\prime}\right)\right],
\end{aligned}
$$

where $A=1 /(1+r)$ and $B=r /(1+r)$. 

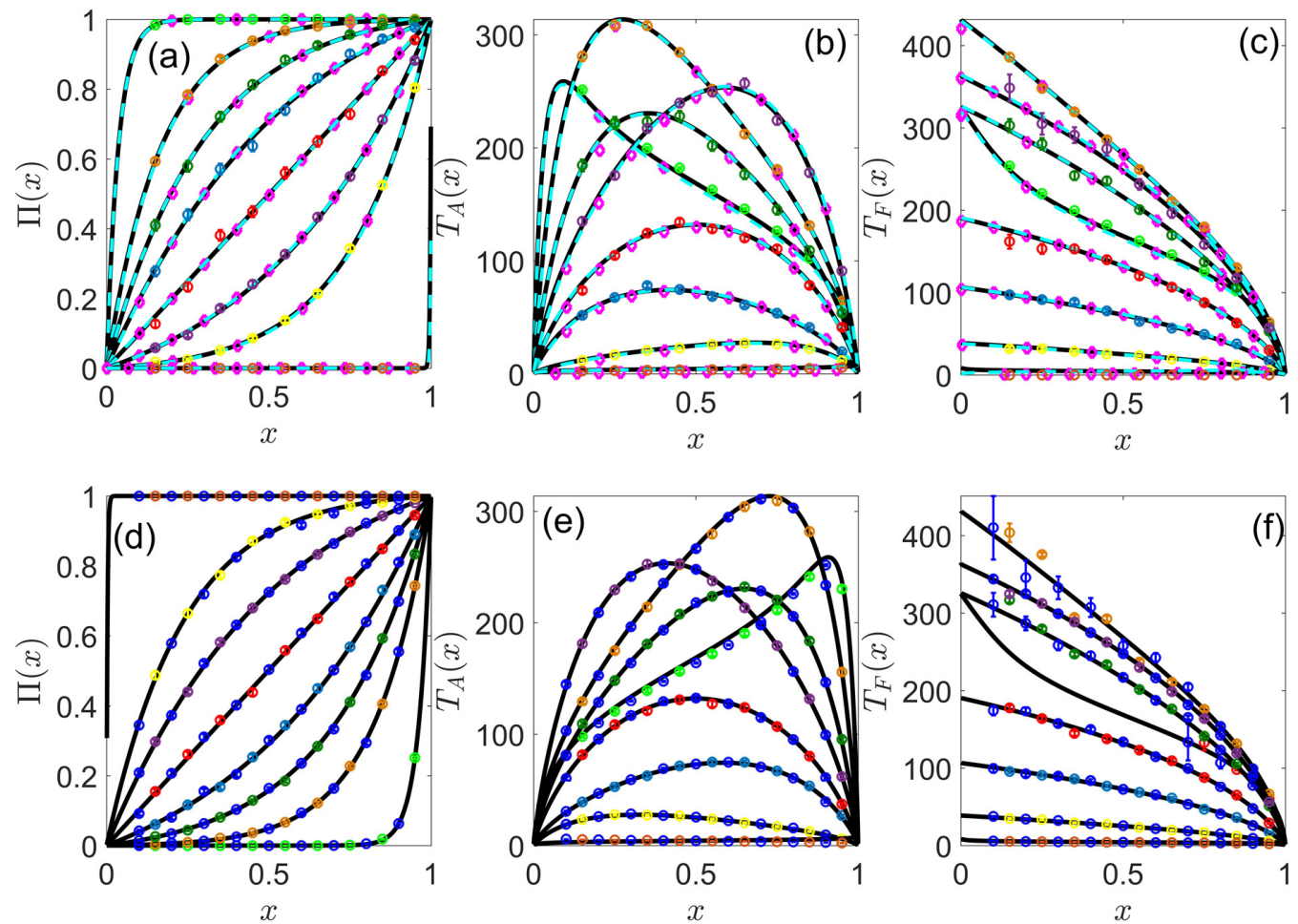

FIG. 2. $\Pi(x)$ [panels (a) and (d)], $T_{A}(x)$ [(b) and (e)], and $T_{F}(x)$ [(c) and (f)] as a function of $x$ for various values of $N, \tau, r$ and selection coefficients. Results for stochastic environment are presented in panels (a)-(c), while the results for periodic variations are shown in panels (d)-(f). For each set of parameters, the solid black line represents the relevant analytic prediction [Eq. (5) for $\Pi$, (19) for $T_{A}$, and (20) for

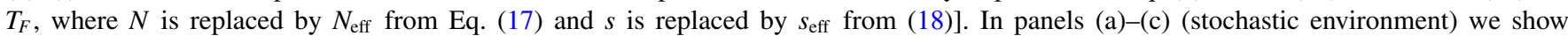
numerical solutions of a Markov matrix model with local (dashed cyan curves) and global (magenta diamonds) competition, together with MC simulation results for local competition (colored circles, plotted with one std error bars that are usually too small to be seen). In panels (d)-(f) (periodic environment), analytic results are compared with MC simulations for local (colored circles) and global (blue circles) competition. Our numerical procedures are explained in Appendix B. Parameters ranges are $N \in[1000,4000], r \in[0.2,0.75]$, and $\tau \in[0.05,5]$; the details for each curve are listed in Appendix A. Parameter set 1 (see Appendix A) is missing in panel (f), since the chance of fixation is too small. The red lines were obtained for combinations of $s, s_{d}$, and $s_{g}$ that yield $s_{\text {eff }}=0$.

Next one would like to replace the sums over many destinations in the last two terms of Eq. (13) by jumps to two possible destinations: one is the mean plus the standard deviation and the other is the mean minus the standard deviation,

$$
\begin{aligned}
& \sum_{x^{\prime}} P_{x \rightarrow x^{\prime}}^{\text {growth }} \Pi\left(x^{\prime}\right) \approx \frac{1}{2}\left[\Pi\left(x+s_{g} x(1-x)(1-r)+\sqrt{\frac{x(1-x)(1-r)}{N}}\right)+\Pi\left(x+s_{g} x(1-x)(1-r)-\sqrt{\frac{x(1-x)(1-r)}{N}}\right)\right], \\
& \sum_{x^{\prime}} P_{x \rightarrow x^{\prime}}^{\text {decline }} \Pi\left(x^{\prime}\right) \approx \frac{1}{2}\left[\Pi\left(x+s_{d} x(1-x)+\sqrt{\frac{x(1-x)(1-r)}{N r}}\right)+\Pi\left(x+s_{d} x(1-x)-\sqrt{\frac{x(1-x)(1-r)}{N r}}\right)\right] .
\end{aligned}
$$

Plugging Eq. (14) and the value of $\eta$ as defined in Eq. (12) into (13) and expanding all functions to second order in $1 / N$ and to first order in selection terms yields

$$
\begin{aligned}
& \left(\frac{1}{2 r}+\frac{1-r}{4 \tau r}\right) \frac{\Pi^{\prime \prime}(x)}{N^{2}} \\
& \quad+\left(\frac{s}{1+r}+\frac{s_{g}(1-r)+s_{d}}{2 \tau(1-r)}\right) \frac{\Pi^{\prime}(x)}{N}=0 .
\end{aligned}
$$

In Eq. (15) we assumed that jumps are relatively rare and $\eta \ll 1$ so $1-\eta \approx 1$; otherwise, the effect of equilibrium competition is negligible in comparison to the effect of the jumps. Note that the condition $s_{d}<(1-r) / r$ ensures that in the limit $r=1$ the contribution from $s_{d}$ vanishes.

$$
\begin{aligned}
& \left(\frac{1}{2 r}+\frac{1-r}{4 \tau r}\right) \frac{T_{A}^{\prime \prime}(x)}{N^{2}}+\left(\frac{s}{1+r}+\frac{s_{g}(1-r)+s_{d}}{2 \tau(1+r)}\right) \frac{T_{A}^{\prime}(x)}{N} \\
& =-\frac{1}{N(1+r) x(1-x)}
\end{aligned}
$$
yield

Similar considerations for $T_{A}(x) \equiv\left[T_{A}^{+}(x)+T_{A}^{-}(x)\right] / 2$ 


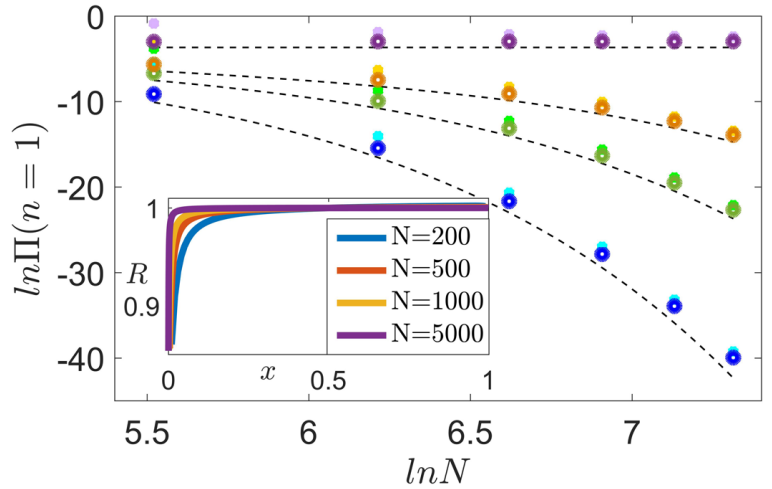

FIG. 3. In the inset we present $R$ vs $x$, where $R$ is the ratio between our analytic expression for $\Pi(x)$ [Eq. (5) with the $N_{\text {eff }}$ given in (17)] and its numerically calculated value for stochastic environment obtained from inversion of the Markov matrix (see Appendix B). Parameters are $r=\tau=0.5$ and $s_{g}=s_{d}=0$. Different values of $N$ were used (see legend), where the value of $s N$ is kept constant $(s N=-0.1)$, so the analytic prediction is $N$ independent. Clearly, the ratio becomes closer and closer to one as $N$ increases, meaning that the quality of our approximation improves with $N$. The results for a single mutant $(n=1$ or $x=1 / N$ ) show the slowest convergence. In the main figure this "worst case scenario" is considered. The logarithm of $\Pi(n=1)$ is plotted against $\ln N$, and the analytic prediction (dashed black lines) is compared with the numerical results for the local (light circle) and global (dark crosses) competition models. All the results were obtained for $\tau=0.1$ and $r=0.5$, and $s_{g}=s_{d}=0$. Markers with different colors stand for $s=-0.2$ (blue). $s=-0.1$ (green), $s=-0.05$ (brown), and $s=0.2$ (purple).

The last term in Eq. (16) reflects the mean time for a significant step, which is $1 / 2 N x(1-x)$ with probability $1 /(1+r)$ and $1 / 2 r N x(1-x)$ with probability $r /(1+r)$.

Comparing Eq. (15) with Eq. (3) and Eq. (16) with Eq. (4), one finds that the solution still has the form of the solution in static environment. Accordingly, one can implement the static environment formulas [like Eq. (5) above] to obtain the $\Pi(x)$, $T_{A}(x)$, and $T_{F}(x)$, by replacing the value of $N$ and $s$ by their effective counterparts

$$
N_{\text {eff }}=N \frac{4 r \tau}{(1+r)(2 \tau+1-r)}
$$

and

$$
s_{\mathrm{eff}}=s+\frac{s_{g}(1-r)}{2 \tau}+\frac{s_{d}}{2 \tau} .
$$

These formulas allow us to calculate the chance of fixation, time to absorption, and time to fixation, using the expressions that were derived for fixed environmental conditions. Before doing that, we would like to discuss the range of applicability of this derivation.

Since our expressions for $N_{\text {eff }}$ and $s_{\text {eff }}$ were derived using the continuum (diffusion) approximation, the agreement be- tween the analytic formula and the numerical results becomes better as $N$ grows, as demonstrated in Fig. 3.

\section{Universality: Local-global and stochastic-periodic equivalence}

As explained in the Introduction, the derivation of (17) and (18) is based on three assumptions. We assumed that the number of up-down flips before fixation is large (when the chance of fixation to take place during a single sweep is large, $\Pi$ depends not only on $x$ but also on the initial state of the environment), that selection parameters are small (we neglected $s^{2}$ terms), and that the diffusion approximation holds ( $N$ and $r N$ are large, so $\Pi$ is relatively smooth over the integers).

Importantly, when these assumptions hold the results are universal, i.e., they do not depend on many details of the model (see below). So far we have analyzed only the case of stochastic variations and local equilibrium competition, but our results turn out to be valid as well for periodic variations and/or global competition.

This amazing property is demonstrated in panels (a) and (d) of Fig. 2. The numerical results (obtained by inverting a Markov matrix or from direct Monte Carlo simulations, as explained in Appendix B) show perfect agreement with the analytic predictions for deleterious, beneficial, and neutral mutants, under global and local competition and in periodic or stochastic environment.

The equivalence of stochastic and periodic variations (when the parameters of the process are calibrated appropriately, as done here) appears to be a generic feature of the approximation used. When the diffusion approximation holds, each "elementary" event (rapid growth, rapid decline, equilibrium dynamics over $\tau$ generations) contributes a given amount of mean change in $x$ to the coefficient of $\Pi^{\prime}(x)$ (or $T_{A}^{\prime}$, etc.), and given variance to the coefficient of $\Pi^{\prime \prime}(x)$. Since we kept only terms that are linear in $s$ (or $s_{d}$ or $s_{g}$ ) and in $1 / N$, each elementary event may split into two or more subevents without changing the corresponding BKE. In the next section, we implement this property when one cannot obtain an exact analytic solution to the contribution of each microstate.

\section{Mean time to absorption and mean time to fixation}

The same diffusion approximation technique allows one obtain the mean time to absorption $T_{A}(x)$ and the mean time to fixation $T_{F}(x)$ using the static environment formulas with $N_{\text {eff }}$ and $s_{\text {eff }}$ as defined in Eqs. (17) and (18). Expressions for $T_{A}(x)$ and $T_{F}(x)$ in static environment were presented in [28] and in Appendix $\mathrm{C}$ we derive simpler expressions for these quantities, using the exponential integral $E_{i}(x)=$ $-\int_{-x}^{\infty} d t \exp (-t) / t$. These are

$$
T_{A}(x)=C_{2}+C_{1} e^{-s N x}+\frac{1}{s}\left(e^{-s N x} E_{i}(s N x)-e^{s N(1-x)} E_{i}[-s N(1-x)]+\ln \left[\frac{1-x}{s N x}\right]\right)
$$


and

$$
\begin{aligned}
T_{F}= & \frac{C_{4}+C_{3} e^{-s N x}+\frac{1}{s}\left(e^{-s N x} E_{i}(s N x)-e^{s N(1-x)} E_{i}[-s N(1-x)]+\ln \left[\frac{1-x}{s N x}\right]\right)}{\Pi(x)} \\
& +\frac{E_{i}[-s N x]-e^{-s N} E i[s N(1-x)]-e^{-s N x} \ln [x /(1-x)]}{s N \Pi(x)} .
\end{aligned}
$$

The constants $C_{1} \ldots C_{4}$ are given in Appendix C. Panels (b),(c) and (e),(f) of Fig. 2 demonstrate the agreement between these formulas (with $N \rightarrow N_{\text {eff }}$ and $s \rightarrow s_{\text {eff }}$ ) and the numerical result for $T_{A}$ and $T_{F}$ in different scenarios.

\section{GRADUAL TRANSITIONS:} A SEMIANALYTIC APPROACH

Until now we considered only two-state systems, in which the total population $N$ may take either of two values and the jumps between them are discontinuous and abrupt. In experimental and natural situations one expects different dynamics. By and large, the changing environmental conditions (for example, increase in precipitation or a draught) affect the potential number of individuals that a given area may sustain, and these changes, as well as the response of the population size $N$ to the variations, are gradual. For example, in standard dilution experiments $r N$ individuals are transferred to the next test tube or petri dish (this is a sharp dilution step with dilution factor $r$ ) but then it takes a few generations (doubling times) until the population reaches the potential carrying capacity of the new environment, where in general during this period the growth is exponential. In nature, one may imagine changes in the potential carrying capacity $K(t)$ after which the total population size $N(t)$ decreases or increases logistically towards the moving target $K(t)$ and so on [31].

In the general scenario many $N$ states (not only two) are allowed, and correspondingly there are many types of jumps (changes of population fraction $x$ in response to environmental variations) and steps (changes in $x$ at fixed $N$ due to competition). Still, as explained above, one may define an effective population size parameter $N_{\text {eff }}$ and an effective selection parameter $s_{\text {eff }}$. As before, an appropriate $s_{\text {eff }}$ reflects the sum over the contributions of all types of jumps and steps to the mean displacement $\Delta x$, and $N_{\text {eff }}$ is determined by the sum of the corresponding contributions to the variance of $\Delta x$. The contribution of each microscopic process has to be weighted by its relative rate of occurrence. An analytic solution in that case requires a tedious (and in many cases impractical) bookkeeping of all possible microstates visited during the process.

To overcome this difficulty, we implement here a semianalytic approach. Since stochastic and periodic processes yield (to first order in $s$ ) the same effective parameters as explained above, we focus on a single cycle of a periodic process and calculate numerically, by averaging over many runs, its contribution to the mean and to the variance. Once $s_{\text {eff }}$ and $N_{\text {eff }}$ are calculated, we plug the results into the relevant fixed environment expressions to obtain the chance of fixation or the time to absorption/fixation. This method allows us to predict the results of a very long process from the mean and the variance of $\Delta x$ for a single cycle.
As an example, we have implemented this approach to the case of logistic growth that resembles the one considered in [31]. The potential carrying capacity $K$ is time dependent, and for convenience it jumps between two values, $K_{ \pm}$. In a single cycle of the periodic dynamics, $K$ alternates between the two states with period $2 \tau$; see Fig. 4 . The total population $N$ responds logistically to $K$ :

$$
\frac{d N}{d t}=\lambda N\left(1-\frac{N}{K(t)}\right) .
$$

The parameter $\lambda$ sets the rate in which $N$ responded to $K$ changes. When $\lambda \tau \gg 1$ the dynamics is close to the twostate system considered before, since $N$ tends to stick to the instantaneous value of $K$. On the other hand, when $\lambda \tau \ll 1$ the value of $N$ fluctuates only weakly around the harmonic mean $2 K_{+} K_{-} /\left(K_{+}+K_{-}\right)$[31].

In our individual-based numerics, after each dual time is incremented by $d t=1 / N(t)$, the new value of $N$ is calculated from Eq. (21) using its exact solution,

$$
N(t+d t)=\frac{N(t) K e^{\lambda d t}}{K-N(t)\left(1-e^{\lambda d t}\right)} .
$$

If $\Delta N=N(t+d t)-N(t)>1, q$ individuals are added, where $q$ is the nearest integer less than or equal to $\Delta N$.

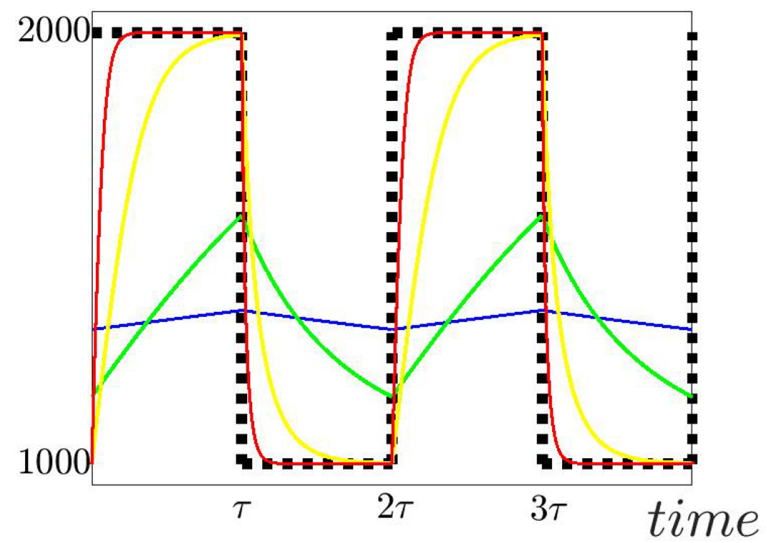

FIG. 4. Population size, $N(t)$, as a function of time, in fluctuating environment with logistic growth [Eq. (21)]. The potential carrying capacities are $K_{+}=2000$ and $K_{-}=1000$ (dotted black lines) and the system switches between these two states every $\tau$ generation (here $\tau=1$ ). The deterministic value of $N$ converges to an orbit $N(t)$. At $t=0$ (where the system jumps to $\left.K=K_{+}\right) N(0)=\{[1+$ $\left.\exp (\lambda \tau)] K_{+} K_{-}\right\} /\left[\exp (\lambda \tau) K_{+}+K_{-}\right]$and the rest of the periodic orbit is given by Eq. (22). When $\lambda \tau \gg 1$, the $N$ orbit traced quite closely the $K$ profile (red, $\lambda=25$ ). As $\lambda \tau$ decreases, population fluctuations flatten out until (at $\lambda \tau \rightarrow 0)$ they converge to $\left(2 K_{+} K_{-}\right) /\left(K_{+}+K_{-}\right)$. The yellow, green, and blue lines are the stable orbits for $\lambda=6$, $\lambda=1$, and $\lambda=0.1$, correspondingly. 

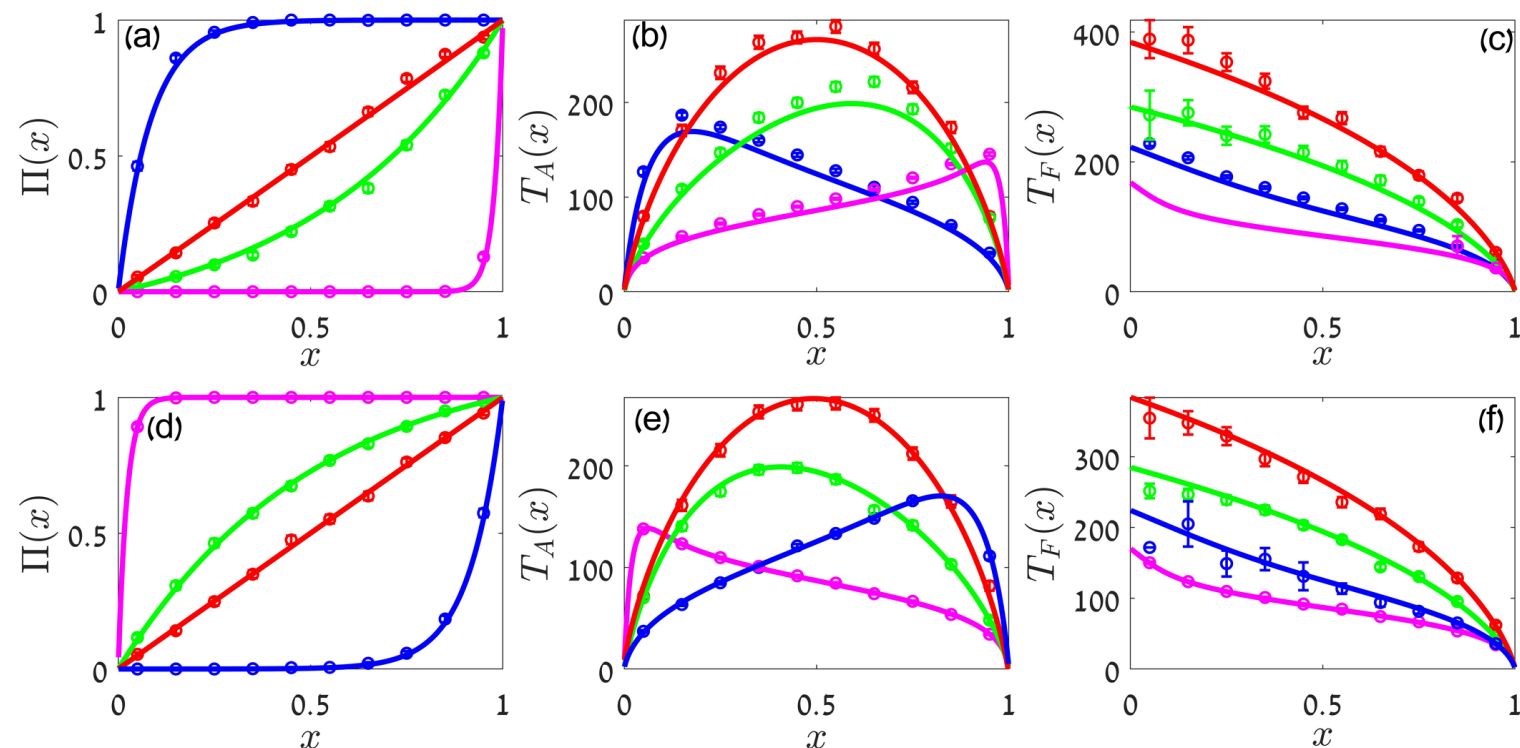

FIG. 5. $\Pi(x)$ [panels (a) and (d)], $T_{A}(x)\left[(\mathrm{b})\right.$ and (e)], and $T_{F}(x)\left[(\mathrm{c})\right.$ and (f)] as a function of $x$ for various values of $K_{+}, K_{-}, \lambda, \tau$, and selection coefficients. Results for stochastic environment are presented in panels (a)-(c), while the results for periodic variations are shown in panels (d)-(f). For each set of parameters, the solid line represents the semianalytic prediction, based on Eqs. (5), (19), and (20), where $N$ and $s$ are replaced by their effective values. $N_{\text {eff }}$ and $s_{\text {eff }}$ were obtained from a one-cycle numerical experiment (the number of trials must be large enough to provide stable mean and variance of $\Delta x$; for the parameter sets at hand we run $10^{6}$ trials) as described in the main text; see Eq. (23). These semianalytic predictions are compared with the outcomes of full Monte Carlo simulations (circles, with one standard deviation error bars), in which the simulation runs from a given initial condition until fixation or extinction (averaged over 1000 runs). Universality implies that periodic and stochastic simulations yield the same results for the same sets of parameters. To avoid duplications of graphs, we invert the values of all selection parameters when crossing from a given stochastic system to its periodic counterpart. The stochastic parameters are $K_{+}=4000, K_{-}=400, \lambda=6, \tau=0.1$, and $s=-0.025, s_{g}=0.0125$, and $s d=0$ (blue); $K_{+}=6000, K_{-}=600, \lambda=12, \tau=0.02$, and $s=0, s_{g}=0$, and $s d=0$ (red); $K_{+}=6000, K_{-}=600, \lambda=16, \tau=0.02$, and $s=-0.00025, s_{g}=-0.00125$, and $s d=-0.001875$ (green); $K_{+}=6000, K_{-}=1500, \lambda=16, \tau=0.02$, and $s=-0.01875, s_{g}=0$, and $s d=-0.025$ (magenta). The chance of fixation [panel (a)] for the magenta curve is vanishingly small (between $10^{-20}$ and $10^{-10}$ for $x<0.8$ ) so we cannot compare the $T_{F}$ predictions with MC simulations in panel (c).

The share of each of the two species in these $q$ slots is determined, as before, by $B_{q}\left[x+s_{g} x(1-x)\right]$. If $\Delta N=N(t+$ $d t)-N(t)<-1,|q|$ individuals are subtracted, where $q$ is the nearest integer greater than or equal to $\Delta N$. In that case each wild type individual survives with probability $\rho=$ $1-q / N(t)$ and a mutant individual survives with probability $\rho^{1+b}$, where $b=\ln \left(1+s_{d}\right) / \ln \left(K_{-} / K_{+}\right)$. (The goal of this parametrization is to make sure that the overall strength of selection when the system declines from $K_{+}$to $K_{-}$is equal to $s_{d}$ that we defined in the last section for sharp jumps.)

Our seminumeric approximation is based on many runs of a single cycle of the periodic dynamics. First we calculated analytically the profile $N(t)$ during a single period. Starting from $N(t=0)$, Eq. (22) provides $N(t=\tau)$ where $K=K_{+}$. A second iteration, using $N(t=\tau)$ as the initial condition and Eq. (22) with $K=K_{-}$, yields $N(t=2 \tau) . N(t=0)$ of the stable orbit is than obtained from the requirement $N(0)=$ $N(2 \tau)$ (see Fig. 4).

The second, numerical step involves many runs of the individual-based model, starting at the calculated value of $N(0)$ and letting the system run for a period of $2 \tau$, where the initial fraction of focal species individuals in the total population is $x(t=0)=n / N(0)$. For each run, $x(t=2 \tau)$ is saved, and the values of $\mu=\mathbb{E}[x(2 \tau)-x(0)]$ and its variance $\sigma^{2}=\mathbb{E}\left[\{x(2 \tau)-x(0)\}^{2}\right]-\mu^{2}$ are obtained from the average over many runs. It turns out that both $\Delta x$ and the variance scale like $x(0)[1-x(0)]$ (the expected behavior for the leading correction in small $s, s_{g}$, and $s_{d}$ ). We then define

$$
s_{\text {eff }}=\frac{\mu}{2 \tau x(1-x)}, \quad N_{\text {eff }}=\frac{4 \tau x(1-x)}{\sigma^{2}},
$$

and calculate $\Pi, T_{A}$, and $T_{F}$ by plugging these values into Eqs. (5), (19), and (20). Some results are depicted in Fig. 5, and one sees a perfect agreement with Monte Carlo simulations.

Note that this semianalytic method admits a selfconsistency requirement, as the values of $s_{\text {eff }}$ and $N_{\text {eff }}$ must be independent of the value of $x(0)$. Our small $s$ approximation is thus (numerically) controlled, and its violation may be identified at this step - if the values of these parameters depend significantly on $x(0)$, one has to pursue the higher-order terms.

\section{DISCUSSION}

Through this paper we presented and examined a methodology aimed at calculating important quantities (chance of fixation, time to fixation, or absorption) for populations in fluctuating environment. To first order in the selection parameters $\left(s, s_{g}\right.$, and $\left.s_{d}\right)$ the diffusion approximation yields a family of backward Kolmogorov equations, like Eqs. (3) and (4). The only effect of the environmental fluctuations is the replacement of $N$ and $s$ by their effective values. 
These effective parameters, $s_{\text {eff }}$ and $N_{\text {eff }}$, reflect the mean frequency displacement $\Delta x$ and its accumulated variance, both measured per one generation. Here we implemented an ergodic approach and replaced the mean and the variance for a given trajectory by the corresponding quantities for an ensemble of trajectories, by calculating the relative contribution of each microstate to the relevant quantities. For sharp transition models, where the number of microstates is small, we calculated analytically these effective parameters by monitoring the contribution of each microstate. This method may be used in other cases, like dilution experiments where the growth phase is a simple exponential, but becomes very difficult when the system visits many microstates. To overcome that, we presented the semianalytic approach described in Sec. III. Such an ergodic approach is feasible, as stated in the Introduction, only if the trajectories are long enough (annealed), which in general translates to the self-consistency condition $T_{F} \gg \tau$.

Our technique relays on an additional condition: commutativity. As long as one neglects $O\left(s^{2}\right)$ and higher order terms, the contributions of each microstate are independent of its place in the chain of events. As a result, the overall answer depends only on the prevalence of each type of event and not on their order. Accordingly, two systems (one periodic and one stochastic, or one with global competition and one with local competition) yield the same effective parameters if their microscopic dynamics is calibrated appropriately. This universality facilitated our calculations: in the second section we solved for stochastic dynamics with local competition and implemented the results to periodic and local competition systems; in the third section we used $N_{\text {eff }}$ and $s_{\text {eff }}$ as obtained numerically for a periodic system and predict the outcome of its stochastic counterpart.

The generally applicable semianalytic approach of Sec. III has a few advantageous features. First, for the first order in $s$ approximation to be valid, the mean and the variance of $\Delta x$ must scale with $x(1-x)$, while if $O\left(s^{2}\right)$ corrections are important this property is lost. Therefore, the first order approximation is controlled by the numerical result. Second, the results obtained for a single cycle allow one to predict the outcome of a whole process that may be arbitrarily long. Here we used this feature to facilitate the analysis of numerical simulations, but one may implement the same technique in the laboratory, predicting the results of long experiments over many generations from the outcomes of a few short, single cycle experiments.

When $s^{2}$ or higher contributions are important, the effects of different environmental states are no longer commutative. For example, equilibrium competition through $\tau$ generations and two $\tau / 2$ periods contribute differently to $s^{2}$ terms. Moreover, global and local competition yield different results [21]. These nonlinear effects are important when equilibrium selection changes sign $[20,21,32]$ and control the efficiency of bet-hedging strategies like phenotypic switching [33,34].

In previous works some authors $[35,36]$ address variations in $N$ and $s$ by implementing Haldane's [37] branching process approximation. This method is limited to calculation of $\Pi$ (not $T_{A}$ or $T_{F}$ ), to the regime $x \ll 1$, and to beneficial mutations only. Our technique is not subject to all these restrictions.
A completely different approach was taken recently by Wienand and co-workers $[31,38]$, who calculated the probability of ultimate fixation for a deleterious mutant in a fluctuating environment (their "pure resource competition scenario"). Wienand et al. considered a logistic growth model that allows for one selection parameter $s$, which reflects the different duplication rates of the two species. As a result, their model admits a single dimensionless parameter, the ratio between the persistence time of the environment $\tau$, and the time to fixation that scales like $1 / s$. In their work the chance of fixation was approximated by the integral

$$
\Pi \approx \int d N \Pi(N) P_{s \tau}(N)
$$

where $\Pi(N)$ is the static environment formula, Eq. (5). $P_{s \tau}(N)$ in this formula is the probability to find the system with $N$ individuals, when the mean time between two environmental switches is the dimensionless parameter $s \tau$. We reexamine this formula and discovered that it works quite nicely not only for the chance of ultimate fixation $\Pi$ and negative selection parameter (the case considered in $[31,38]$ ), but also for positive selection and for the time parameters $T_{A}$ and $T_{F}$.

However, our models admit many time scales (different selection parameters and the environmental response parameter $\lambda$, together with $\tau$ ) so there is no single dimensionless parameter. Accordingly, this strategy of replacing $\tau$ in $P_{\tau}(N)$ by the dimensionless parameter is not applicable. As a result, we cannot use the clever ansatz (24) and instead we had to develop a generic diffusion-based approach. The price we had to pay for this reliance on the diffusion approximation is that our theory is limited to the annealed regime where the system visits each state many times before fixation or extinction. Our result must be interpreted accordingly. For example, taking $\tau$ to infinity in our formulas is allowed only if it diverges with $N$ such that $T_{F}$ remains much larger than $\tau$.

Interestingly, once the system reaches the annealed limit in the model considered by Wienand et al., further decrease of $\tau$ does not affect the outcome that sticks to the result at constant population, where this constant corresponds to the harmonic mean of the carrying capacities. Our models, with more degrees of freedom, show quite a rich behavior in the annealed regime.

\section{ACKNOWLEDGMENTS}

We thank B. Good and D. Kessler for helpful discussions. This research was supported by the ISF-NRF Singapore joint research program (Grant No. 2669/17). The work of I.M. was supported by an Eshkol Fellowship of the Israeli Ministry of Science.

\section{APPENDIX A: DETAILED DESCRIPTION OF THE PARAMETER SETS USED IN FIG. 2}

Figure 2 of the main text is reproduced here with a number attached to each data set. Here, in Fig. 6, the parameters are given for each number in Table II. 

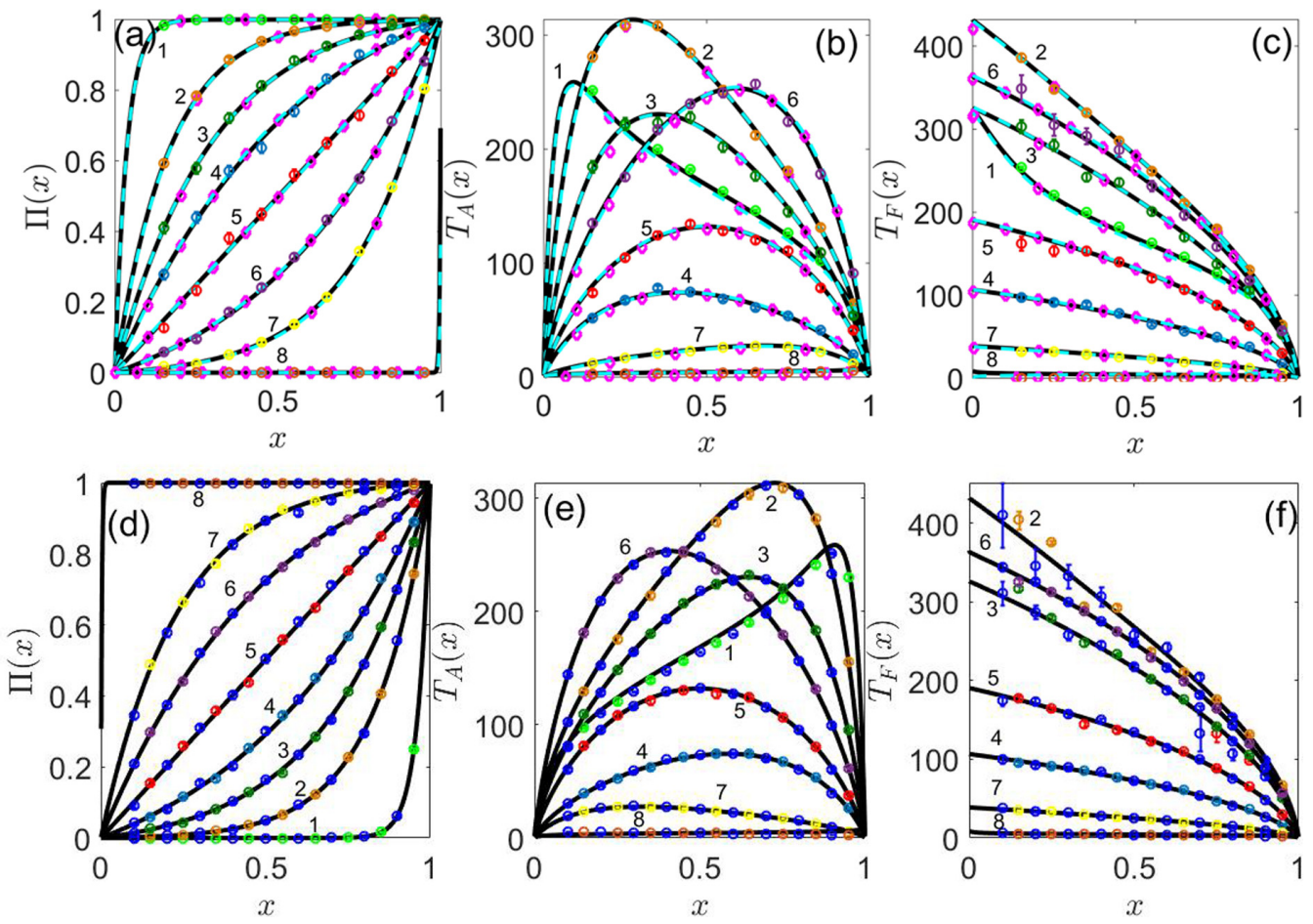

FIG. 6. $\Pi(x)$ [panels (a) and (d)], $T_{A}(x)\left[(\mathrm{b})\right.$ and (e)], and $T_{F}(x)[(\mathrm{c})$ and (f)] as a function of $x$ for various values of $N$. The number attached to each line in panels (a)-(c) corresponds to a different set of parameters, detailed in Table II. Panels (d)-(f) were obtained for exactly the same set of parameters, except that the direction of selection was reversed (i.e., $s \rightarrow-s, s_{g} \rightarrow-s_{g}$, and $s_{d} \rightarrow-s_{d}$ ).

\section{APPENDIX B: NUMERICAL TECHNIQUES}

Through this paper we compared the analytic predictions [Eqs. (5), (19), and (20) of the main text, with $N_{\text {eff }}$ and $\left.s_{\text {eff }}\right]$ with numerical results that were obtained from two types of numerical calculations. Here we provide some details for the numerics.

\section{Markov matrix inversion}

The essence of this technique is explained in Eq. (11) above and in the following text; for more details and examples see Appendix A2 of [39] or Appendix A of [40].

A technical problem appeared when we tried to implement our method to the sharp decline period. If the number of mutant individuals is picked at random from $B_{x N}[r(1+$ $\left.s_{d}\right)$ ] and the number of wild type is picked independently from $B_{(1-x) N}[r]$, the total size of the population after the decline fluctuates around $N r$. To implement our exact numeric technique the total population size after the decline period must be exactly $N r$, so we cannot use two independent binomial trials.

To overcome this difficulty we assumed that, in a decline step,

$$
n \rightarrow r \frac{n^{2}}{N}+s_{d} r n(1-n / N)+B_{\frac{n(N-n)}{N}}[r]
$$

Therefore,

$$
P_{n \rightarrow m}^{\mathrm{decline}}=f_{N x(1-x), r}\left[m-r N x^{2}+s_{d} r N x(1-x)\right] .
$$

When $s_{d}<<1$ this process has the same mean and variance of the two independent binomial trial processes, and since the solution involves only the mean and the variance, it yields the same outcomes.

TABLE II. Parameters: Fig. 2.

\begin{tabular}{lccccccc}
\hline \hline Index & Color & $N$ & $\tau$ & $r$ & $s$ & $s_{g}$ & $s_{d}$ \\
\hline 1 & Light green & 4000 & 1 & 0.25 & 0.01 & -0.03 & 0.05 \\
2 & Light brown & 2500 & 1 & 0.2 & 0.01 & 0 & 0 \\
3 & Dark green & 1000 & 5 & 0.25 & 0.01 & 0.0 & -0.01 \\
4 & Turquoise & 1000 & 0.05 & 0.5 & 0.001 & 0.001 & 0.001 \\
5 & Red & 1000 & 0.1 & 0.5 & -0.002 & 0.01 & -0.0046 \\
6 & Purple & 2000 & 0.1 & 0.5 & -0.005 & 0.000001 & -0.00001 \\
7 & Yellow & 1000 & 0.05 & 0.25 & 0.01 & 0 & -0.01 \\
8 & Dark brown & 1000 & 0.05 & 0.75 & 0 & 0.2 & -0.2 \\
\hline \hline
\end{tabular}




\section{Monte Carlo simulations}

We have implemented direct MC simulations for two reasons. First, the Markov matrix inversion numerics is applicable only in the stochastic case, when per each step there is a finite probability that the system switches from poor to rich state or vice versa. To implement the same matrix technique in the periodic case one must calculate first the corresponding Floquet operator for equilibrium periods of duration $\tau$. Instead, we implemented direct MC simulations.

Secondly, the Markov matrix technique is applicable only if the system jumps between two states, one with exactly $N$ individuals and the other with $r N$. As we have seen above, this restriction is incompatible with the realistic modeling of sharp decline periods, where the chance of each individual to survive is $r$, so we implemented an ad hoc binomial mass function which has the same mean and variance. In the MC simulation, on the other hand, decline involves two independent binomial deviates, one for the mutant and one for the wild type, without any global restriction.

\section{APPENDIX C: DERIVATION OF $T_{A}$ AND $T_{F}$ IN STATIC ENVIRONMENT}

The formulas derived below are our simplified version of the solutions presented in [28], p. 430.

\section{Calculation of $T_{A}$}

To calculate $T_{A}$ in static environmental conditions ( $s$ and $N$ are time independent) one writes the backward Kolmogorov equation (BKE). Starting with $n$ individuals, the time to either fixation or extinction is $T_{n}=\sum W_{n, m} T_{m}+\Delta t$, where $\Delta t$ is the time needed for a step (in our case, $\Delta t=1 / N$ ). The sum is over all possible destination states [in our case, $m \in$ $[n-1, n, n+1]$ and the $W$ 's are the transition probabilities defined in Eq. (10)]. Accordingly,

$$
\begin{aligned}
T_{n}= & 2 x(1-x)\left(\frac{1+s / 2}{2} T_{n+1}+\frac{1-s / 2}{2} T_{n-1}\right) \\
& +[1-2 x(1-x)] T_{n}+\frac{1}{N} .
\end{aligned}
$$

In the continuum limit, $x=n / N$ and $T_{n \pm 1} \approx T(x) \pm$ $T^{\prime}(x) / N+T^{\prime \prime}(x) /\left(2 N^{2}\right)$,

$$
T^{\prime \prime}+s N T^{\prime}=-\frac{N}{x(1-x)} .
$$

The boundary conditions are of course $T(0)=T(1)=0$.

Using the integration factor $\exp (s N x)$ one may find $T^{\prime}$,

$$
T^{\prime}=C e^{-s N x}-N e^{-s N x} E_{i}(s N x)+N e^{s N(1-x)} E_{i}(-s N[1-x]),
$$

where we used the exponential integral

$$
E_{i}(x)=-\int_{-x}^{\infty} d t \frac{e^{-t}}{t} .
$$

Second integration and implementation of the boundary conditions yields Eq. (19) of the main text with

$$
\begin{aligned}
& C_{1}=\frac{-2 e^{s N} \gamma_{E}+e^{2 s N} E_{i}(-s N)+E_{i}(s N)-2 e^{s N} \ln (s N)}{s\left(e^{s N}-1\right)}, \\
& C_{2}=\frac{\gamma_{E}\left(1+e^{s N}\right)-e^{s N}\left[2 \ln (s N)-E_{i}(-s N)\right]-E_{i}(s N)}{s\left(e^{s N}-1\right)} .
\end{aligned}
$$

\section{Calculation of $T_{F}$}

To calculate $T_{F}$ one defines [41] $Q=\Pi \cdot T_{F} \cdot Q$ satisfies

$$
Q^{\prime \prime}+s N Q^{\prime}=-\frac{N}{x(1-x)}+\frac{N e^{-s N x}}{x(1-x)},
$$

with the boundary conditions $Q(0)=Q(1)=0$. Except for the last term, we obtained the same equation as Eq. (C2), meaning that

$$
Q^{\prime}(x)=T_{A}^{\prime}(x)+N \ln \left(\frac{x}{1-x}\right) e^{-s N x} .
$$

Accordingly, one obtains Eq. (20) of the main text with

$$
\begin{gathered}
C_{3}=\frac{-3 \gamma_{E} e^{s N}-3 e^{s N} \ln (s N)-\ln (s N)+e^{s N} \operatorname{Ei}(-s N)+e^{2 s N} \operatorname{Ei}(-s N)+2 \operatorname{Ei}(s N)-\gamma_{E}}{\left(e^{s N}-1\right) s}, \\
C_{4}=\frac{e^{-s N}\left[3 \gamma_{E} e^{s N}+\gamma_{E} e^{2 s N}+2 e^{s N} \ln (s N)+2 e^{2 s N} \ln (s N)-2 e^{2 s N} \operatorname{Ei}(-s N)-e^{s N} \operatorname{Ei}(s N)-\operatorname{Ei}(s N)\right]}{\left(e^{s N}-1\right) s} .
\end{gathered}
$$

[1] R. Lande, S. Engen, and B.-E. Saether, Stochastic Population Dynamics in Ecology and Conservation (Oxford University Press, Oxford, 2003).

[2] T. L. Parsons, C. Quince, and J. B. Plotkin, Genetics 185, 1345 (2010).

[3] N. Takahata, K. Ishii, and H. Matsuda, Proc. Natl. Acad. Sci. USA 72, 4541 (1975).
[4] N. Takahata and M. Kimura, Proc. Natl. Acad. Sci. USA 76, 5813 (1979).

[5] A. O. Bergland, E. L. Behrman, K. R. O'Brien, P. S. Schmidt, and D. A. Petrov, PLoS Genet. 10, e1004775 (2014).

[6] G. Bell, Philos. Trans. R. Soc. London B: Biol. Sci. 365, 87 (2010). 
[7] P. W. Messer, S. P. Ellner, and N. G. Hairston, Jr., Trends Genet. 32, 408 (2016).

[8] C. E. Cáceres, Proc. Natl. Acad. Sci. USA 94, 9171 (1997).

[9] H. E. Hoekstra, J. M. Hoekstra, D. Berrigan, S. N. Vignieri, A. Hoang, C. E. Hill, P. Beerli, and J. G. Kingsolver, Proc. Natl. Acad. Sci. USA 98, 9157 (2001).

[10] E. G. Leigh, J. Evol. Biol. 20, 2075 (2007).

[11] D. R. Hekstra and S. Leibler, Cell 149, 1164 (2012).

[12] M. Kalyuzhny, E. Seri, R. Chocron, C. H. Flather, R. Kadmon, and N. M. Shnerb, Am. Nat. 184, 439 (2014).

[13] M. Kalyuzhny, Y. Schreiber, R. Chocron, C. H. Flather, R. Kadmon, D. A. Kessler, and N. M. Shnerb, Ecology 95, 1701 (2014).

[14] R. A. Chisholm, R. Condit, K. A. Rahman, P. J. Baker, S. Bunyavejchewin, Y.-Y. Chen, G. Chuyong, H. Dattaraja, S. Davies, C. E. Ewango et al., Ecol. Lett. 17, 855 (2014).

[15] V. Mustonen and M. Lässig, Phys. Rev. Lett. 100, 108101 (2008).

[16] E. Huerta-Sanchez, R. Durrett, and C. D. Bustamante, Genetics 178, 325 (2008).

[17] P. Ashcroft, P. M. Altrock, and T. Galla, J. R. Soc., Interface 11, 20140663 (2014).

[18] I. Cvijović, B. H. Good, E. R. Jerison, and M. M. Desai, Proc. Natl. Acad. Sci. USA 112, E5021 (2015).

[19] J. Hidalgo, S. Suweis, and A. Maritan, J. Theor. Biol. 413, 1 (2017).

[20] M. Danino and N. M. Shnerb, J. Theor. Biol. 441, 84 (2018)

[21] I. Meyer and N. M. Shnerb, Sci. Rep. 8, 9726 (2018).

[22] L. Marrec and A.-F. Bitbol, PLoS Comput. Biol. 16, e1007798 (2020).

[23] W. J. Ewens, Mathematical Population Genetics 1: Theoretical Introduction (Springer Science \& Business Media, New York, 2012), Vol. 27.
[24] M. M. Desai, D. S. Fisher, and A. W. Murray, Curr. Biol. 17, 385 (2007).

[25] M. Danino, D. A. Kessler, and N. M. Shnerb, J. Stat. Phys. 172, 126 (2018).

[26] D. A. Kessler and N. M. Shnerb, J. Stat. Phys. 127, 861 (2007).

[27] B. H. Good, M. J. McDonald, J. E. Barrick, R. E. Lenski, and M. M. Desai, Nature (London) 551, 45 (2017).

[28] J. F. Crow, M. Kimura et al., An Introduction to Population Genetics Theory (Burgess Publishing Company, Minneapolis, $\mathrm{MN}, 1970)$.

[29] C. C. Smith and S. D. Fretwell, Am. Nat. 108, 499 (1974).

[30] L. M. Wahl and P. J. Gerrish, Evolution 55, 2606 (2001). Note that the factor $1-r$ was neglected by Wahl and Gerrish, who assumed that $r \ll 1$.

[31] K. Wienand, E. Frey, and M. Mobilia, Phys. Rev. Lett. 119, 158301 (2017).

[32] X. Yi and A. M. Dean, Proc. Natl. Acad. Sci. USA 110, 16945 (2013).

[33] M. Thattai and A. Van Oudenaarden, Genetics 167, 523 (2004).

[34] P. Patra and S. Klumpp, Phys. Biol. 12, 046004 (2015).

[35] S. Engen, R. Lande, and B.-E. Sæther, Genet. Res. 91, 73 (2009).

[36] H. Uecker and J. Hermisson, Genetics 188, 915 (2011).

[37] J. B. S. Haldane, Mathematical Proceedings of the Cambridge Philosophical Society (Cambridge University Press, Cambridge, UK, 1927), Vol. 23, pp. 838-844.

[38] K. Wienand, E. Frey, and M. Mobilia, J. R. Soc., Interface 15, 20180343 (2018).

[39] M. Danino, D. A. Kessler, and N. M. Shnerb, Theor. Popul. Biol. 119, 57 (2018).

[40] Y. Yahalom, B. Steinmetz, and N. M. Shnerb, Phys. Rev. E 99, 062417 (2019).

[41] S. Redner, A Guide to First-passage Processes (Cambridge University Press, Cambridge, UK, 2001). 